\title{
Las especies neotropicales de Proisotoma (Collembola: Isotomidae), con descripción de dos especies nuevas cavernícolas de América
}

\author{
Neotropical species of Proisotoma (Collembola: Isotomidae), with description of two new cave \\ species from America
}

\author{
José G. Palacios-Vargas ${ }^{1 *}$ y Javier I. Arbea ${ }^{2}$ \\ ${ }^{1}$ Laboratorio de Ecología y Sistemática de Microartrópodos, Departamento de Ecología y Recursos Naturales, Facultad de Ciencias, Universidad \\ Nacional Autónoma de México, 04510 México, D. F., México. \\ ${ }^{2}$ Departamento de Biología y Geología, IES Foramontanos, Av.Constitución s/n, 39500 Cabezón de la Sal, Cantabria, España. \\ *Correspondencia: troglolaphysa@hotmail.com
} Resumen. Se describen e ilustran 2 especies nuevas cavernícolas de Proisotoma s. str., P. turikana sp. nov. de Venezuela,
y P. santosorum sp. nov. de México. Además, se proporciona una clave para identificar las especies neotropicales de
Proisotoma sensu lato y géneros cercanos (Ballistura, Folsomides, Guthriella, Isotoma, Mucrotoma, Varisotoma, Scutisotoma).

Palabras clave: Proisotoma, cavernícolas, región neotropical, sistemática, clave.

\begin{abstract}
Two new cave species of Proisotoma s. str. are described and illustrated: P. turikana sp. nov. from Venezuela, and P. santosorum sp. nov. from Mexico. A key for the identification of the Neotropical species of Proisotoma sensu lato and some related genera (Ballistura, Folsomides, Guthriella, Isotoma, Mucrotoma, Varisotoma, and Scutisotoma) is included.
\end{abstract}

Key words: Proistoma, caves, Neotropical region, systematics, key.

\section{Introducción}

En el catálogo de colémbolos de la región neotropical de Mari Mutt y Bellinger (1990) se citan 28 especies del género Proisotoma Börner, 1901: P. alticola (Loksa y Rubio), P. andina Rapoport y Rubio, P. biseta Rapoport, $P$. centralis Denis, $P$. fatonei Rapoport, $P$. fernandeziana Rubio, $P$. filifera Denis, $P$. fitchi Denis, $P$. intermedia Izarra, $P$. intermixta Handschin, $P$. koepckei Winter, $P$. lutii Izarra, $P$. macedoi Winter, $P$. micrura Börner, $P$. minima (Absolon), P. minuta (Tullberg), P. obtusicauda (Schäffer), $P$. oliveirae Deharveng, P. pajonalica Loksa y Rubio, $P$. paronai Börner, $P$. perparva Jackson, $P$. plicicauda Handschin, $P$. ramosi Arlé, $P$. ridleyi Denis, $P$. subminuta Denis, P. tenella (Reuter), P. titusi Folsom y P. troglobia Rapoport y Maño. Con posterioridad se han citado otras 5 especies en esta región: $P$. cf. excavata (Palacios-Vargas, 1990), P. immersa (Palacios-Vargas et al., 2000; Vázquez y Palacios-Vargas, 2004), P. frisoni (Palacios-Vargas y Castaño-Meneses, 2002), P. beta y $P$. cf. bulba (Vázquez y Palacios-Vargas, 2004). P. minuta y una especie inédita

Recibido: 12 septiembre 2008; aceptado: 14 enero 2009 fueron citadas recientemente por Cutz-Pool et al. (2007). Según los criterios de Potapov et al. (2006), algunas de estas especies pertenecen a géneros diferentes: Folsomides Stach, 1922, Ballistura Börner, 1906, Scutisotoma Bagnall, 1949 y otros más, aún por definir (véase discusión al final del trabajo).

En este trabajo se describen 2 nuevas especies de Proisotoma s. str., P. turikana.sp. nov. de cuevas de Venezuela y P. santosorum sp. nov. de cuevas de México.

\section{Materiales y métodos}

Los ejemplares fueron colectados en muestras de guano o detritos de madera en cuevas de Venezuela y de México respectivamente. Se extrajeron por medio del embudo de Berlese-Tullgren $y$ fueron montados entre porta $y$ cubreobjetos para su estudio. Posteriormente se dibujaron con ayuda de cámara lúcida para su descripción. Las mediciones se realizaron con una reglilla en el micrómetro ocular. Los tipos de las nuevas especies están depositados en la colección de Collembola del Laboratorio de Ecología y Sistemática de Microartrópodos (LESM). 


\section{Descripciones}

\section{Proisotoma Börner, 1901}

Diagnosis. Las especies tradicionalmente incluidas en Proisotoma pertenecen a varias líneas evolutivas diferentes y actualmente son objeto de estudio por Potapov et al. (2006). Proisotoma s. str. incluye las Anurophorinae con todos los segmentos abdominales claramente separados y una fúrcula completa pero relativamente corta; manubrio con un par de sedas distales en la cara anterior; dentes con pocas sedas (4-9 anteriores y 3-7 posteriores) extendiéndose las sedas anteriores hasta la base; mucrón con 2-3 dientes y sin lamelas laterales; sensilas de los tergitos abdominales situadas en la fila $p$ de sedas; con un número reducido de microsensilas (10/000 ó 00/ 000); fila de sedas $B$ en los tibiotarsos 1-2 completa. El género es cosmopolita e incluye especies que viven típicamente en restos orgánicos.

Proisotoma turikana sp. nov. (Figs. 1-8)

Longitud 0.9-1.2 mm. Coloración azul. Cuerpo alargado, de la misma forma que $P$. minuta. Revestimiento dorsal y ventral constituido por sedas ordinarias cortas, lisas y puntiagudas, con macrosedas ligeramente diferenciadas en los terguitos abdominales V y VI; sensilas poco diferentes de las sedas ordinarias, son algo más finas; microsensila del terguito torácico II muy corta (Fig. 1). Antenas un poco más largas que la diagonal cefálica. Artejo antenal I con 12 sedas ordinarias, una microseda basal ventral y 2 sensilas bastoniformes ventrales, una gruesa y otra fina. Antenal II con 18 sedas ordinarias, 3 microsedas basales y una seda sensorial fina ventro-distal. Antenal III con 23 sedas ordinarias, 9 sedas sensoriales finas y un órgano sensorial formado por 2 microsensilas internas bastoniformes, 2 sedas sensoriales de guardia largas y finas y 2 microsensilas ventroexternas. Revestimiento de antenal IV abundante, formado por sedas ordinarias y unas 20 sedas sensoriales largas y finas, poco diferenciadas; apicalmente presenta una microseda y un organito muy pequeño; sin vesícula apical excértil (Fig. 3). Cabeza con 5+5 ojos, dentro de una mancha ocular negra. Órgano postantenal ovalado, 2, 5 veces más largo que el diámetro de una corneola (Fig. 4). Palpo maxilar simple, con 4 sedas sublobulares (Fig. 5). Quetotaxia clipeolabral 3/ 5, 5, 4, insertándose estas 4 últimas sedas en papilas desarrolladas; margen anterior del labro sin estructuras particulares (Fig. 6).

Quetotaxia sensorial sobre los terguitos:

\begin{tabular}{llllllll}
\multicolumn{3}{c}{ Tórax } & \multicolumn{7}{c}{ Abdomen } \\
Sensila & II & III & I & II & III & IV & V \\
accp & 2 & 2 & 3 & 3 & 3 & 3 & 4 \\
al & $2+1 m s$ & 2 & 0 & 0 & 0 & 0 & 0
\end{tabular}

Revestimiento ordinario abundante (Fig. 1). Terguito abdominal VI con las sedas dorsales impares a0 y $\mathrm{mo}$. Ventralmente se observan: 4+4 sedas a lo largo de la línea ventral cefálica; esternitos torácicos I, II, III con $0,0,1$ pares de sedas; tubo ventral con 5-6 sedas posterobasales y 4-5+4-5 sedas laterodistales; abdominal II con 3+3 sedas mediales. El esternito abdominal IV lleva 19-20 sedas en la subcoxa anterior y 8-9 en la subcoxa posterior (Fig. 2). Fúrcula similar a la de $P$. minuta; manubrio con 20-22+2022 sedas posteriores y $1+1$ sedas anteriores más gruesas; dente no anillado, con 6 sedas en la cara anterior (1 en la mitad basal, $2+2$ mediales y 1 en la parte distal) y 7 sedas en la cara posterior ( 4 sedas basales, 2 submediales y 1 subapical) (Fig. 7); relación manubrio/ dente/ mucrón: 5, 4/ 3, 7/ 1; mucrón tridentado. Tenáculo con 4+4 dientes y 1 seda en el cuerpo.Ungues sin dientes internos, ni laterales; fino y alargado, relación longitud de su borde interno: anchura de la base =3-3, 5. Apéndice empodial con una lamela interna redondeada, bien desarrollada y sin dientes, alcanza el 60-70\% de la longitud del borde interno de la uña. Tibiotarsos I, II, III con 21, 22, 24-25 sedas, respectivamente, de las que $1,1,1$ son sedas dorsales largas y ligeramente capitadas (Fig. 8).

Quetotaxia de las patas:

Subcoxa 1 Subcoxa 2 Coxa Trocánter

$\begin{array}{lcccc}\text { Pata I } & 0 & 1 & 4 & 8-9 \\ \text { Pata II } & 3-5 & 6 & 9-10 & 8-9 \\ \text { Pata III } & 7-8 & 7-8 & 9-10 & 7 \\ & \text { Fémur } & & \text { Tibiotarso } & \\ & 16 & & 21 \\ & 16 & & 21 & \\ & 19 & & 26\end{array}$

Etimología. El nombre hace referencia a la región en la que ha sido encontrada la especie: Mesa Turik, Venezuela.

\section{Comentarios taxonómicos}

Poco después de su recolección, esta especie se citó como Proisotoma sp. (Viloria et al., 1992). Pertenece al mismo grupo que $P$. minuta, la especie tipo del género. Este grupo de especies se caracteriza por presentar mucrón tridentado, órgano postantenal ovalado, uña sin dientes, el manubrio más largo que el dente, $1+1$ sedas anteriores en el manubrio y pocas sedas en el dente (5-6 sedas anteriores y 5-7 posteriores). En la región neotropical, pertenecen a este grupo con seguridad las siguientes especies: $P$. minuta (Tullberg, 1871), P. subminuta Denis, 1931, $P$. minima (Absolon, 1901), P. oliveirae Deharveng, 1984 


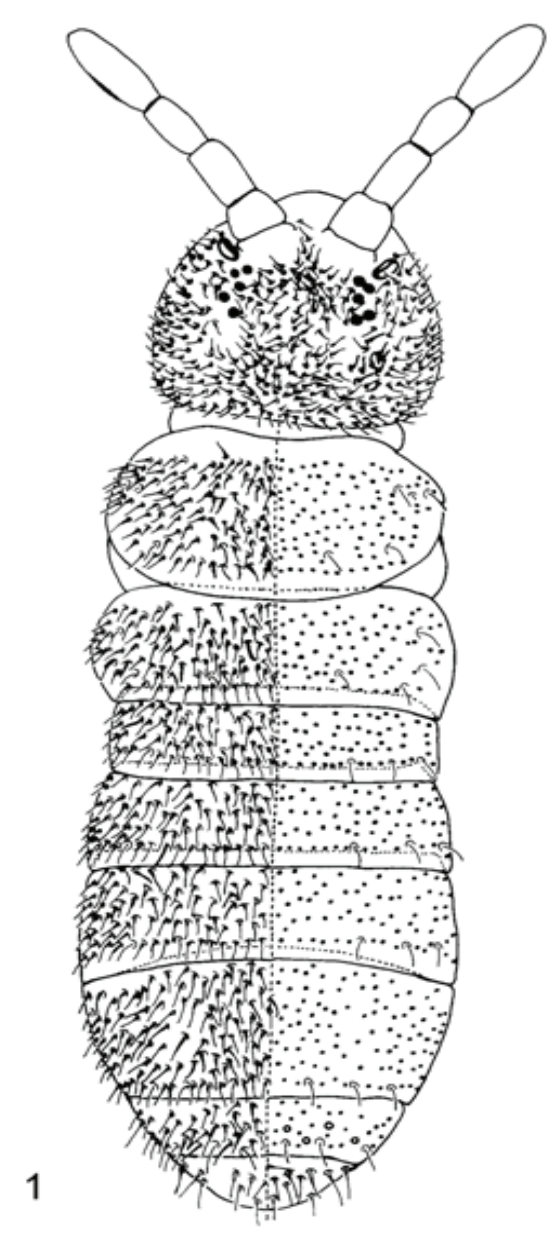

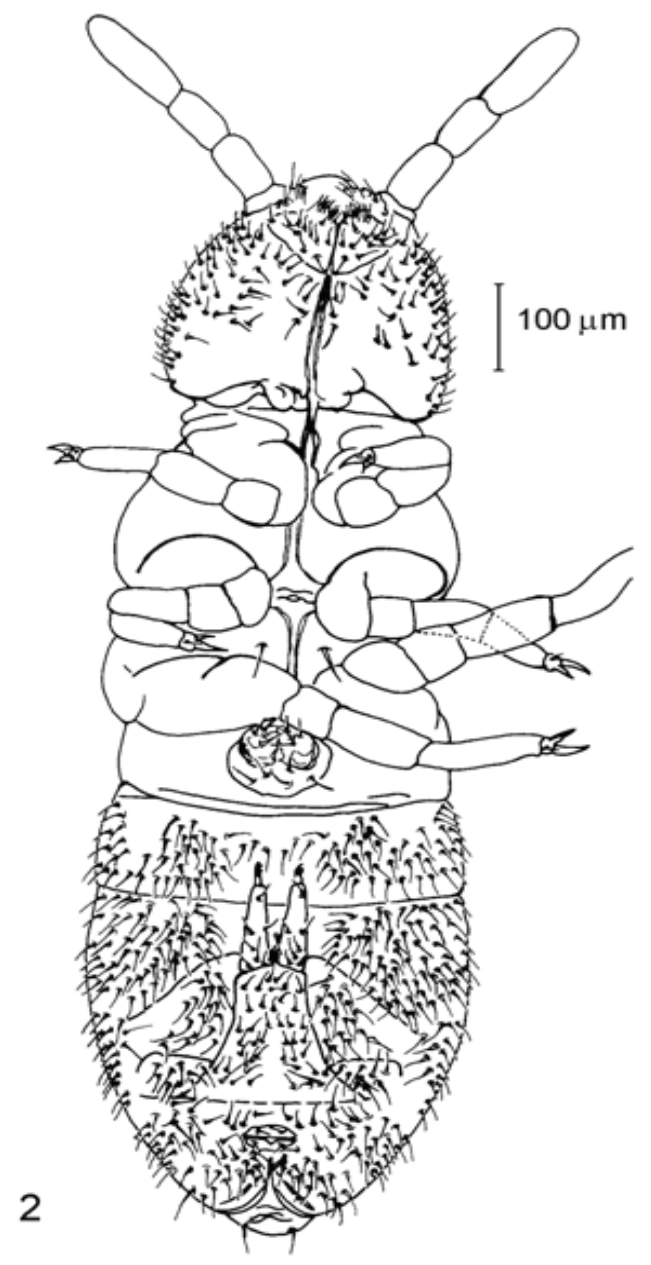
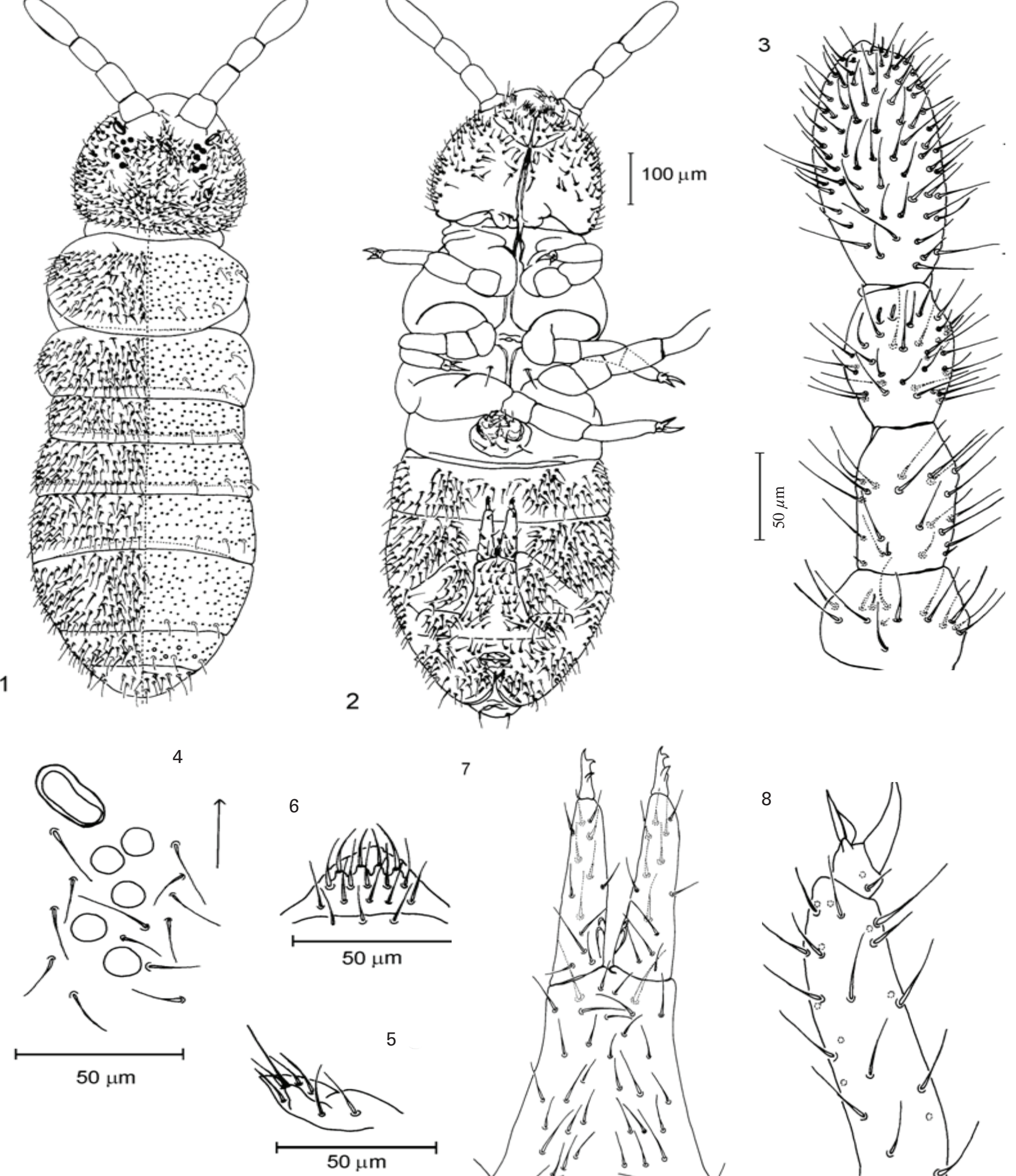

Figuras 1-8. Proisotoma turikana sp. nov. 1, quetotaxia dorsal; 2, quetotaxia ventral; 3 , antena; 4 , ojos y órgano postantenal; 5, palpo maxilar; 6, labro; 7 , fúrcula, y 8 , tibiotarso y uña III.
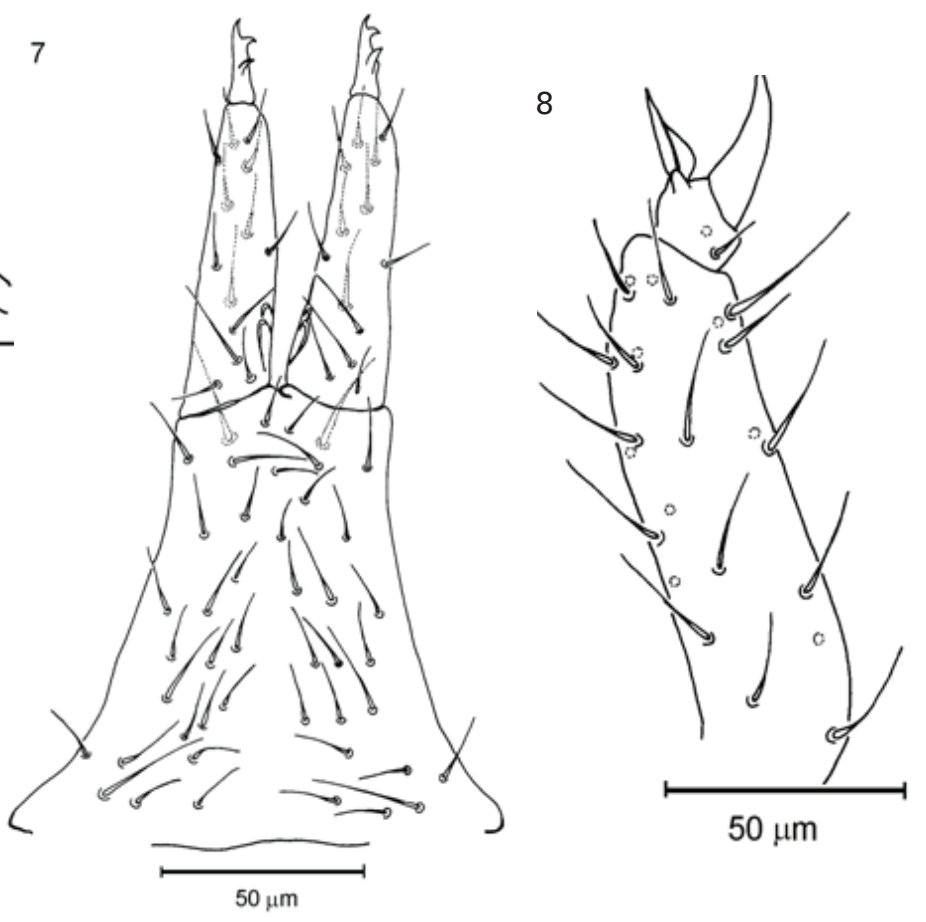


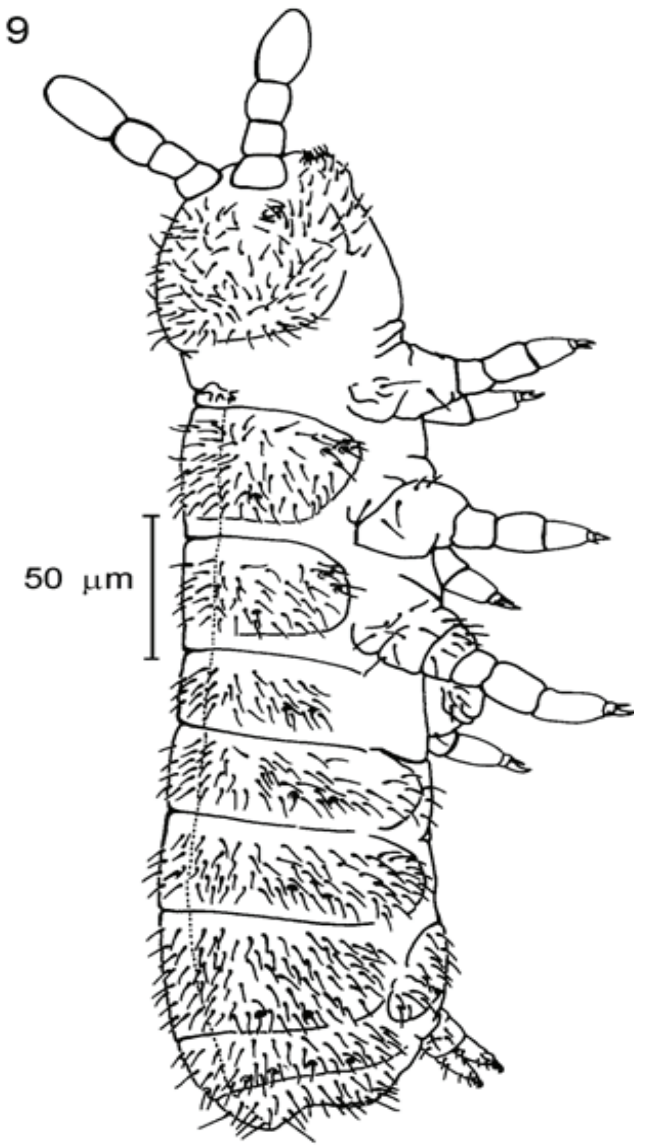

12

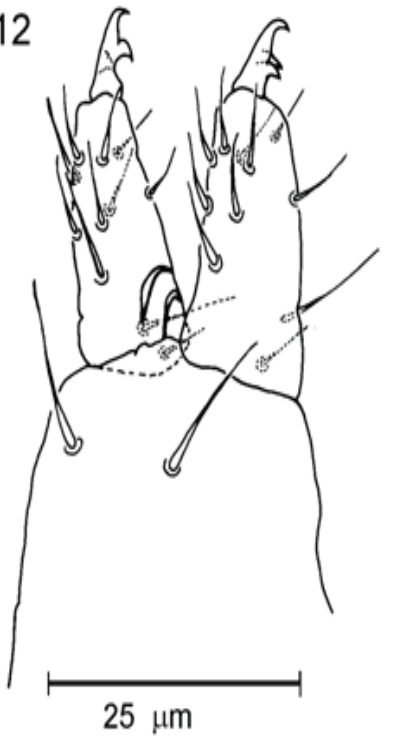

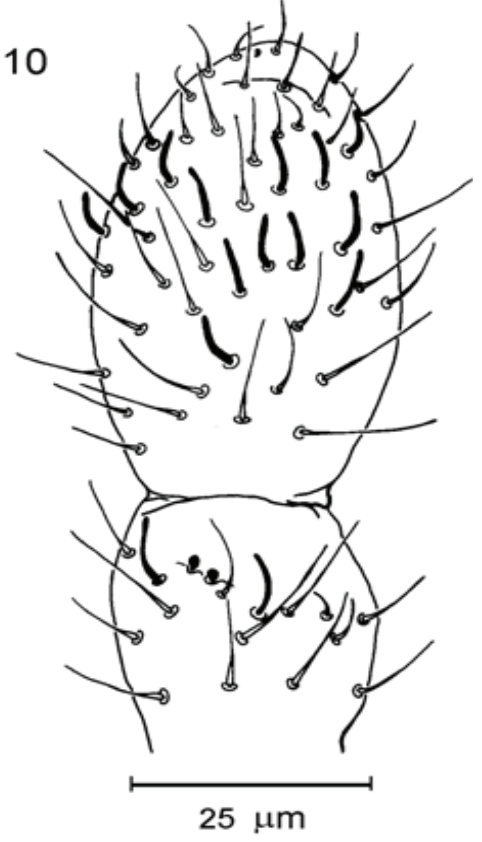

$25 \mu \mathrm{m}$

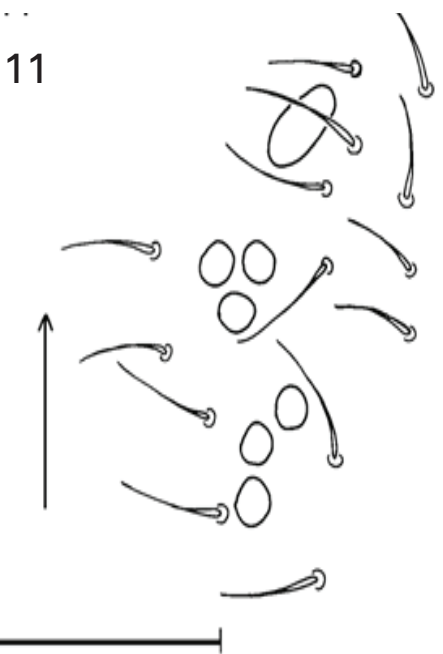

$25 \mu \mathrm{m}$

Figuras 9-13. Proisotoma santosorum sp. nov. de México. 9. Quetotaxia dorsal y ventral. 10. Artejos antenales III y IV. 11. Ojos y órgano postantenal. 12. Fúrcula. 13. Tibiotarso y uña III.

y $P$. turikana sp. nov. y $P$. santosorum sp. nov. De todas éstas , $P$. turikana es más parecida a $P$. minuta. Ambas poseen un número similar de sedas en los esternitos torácicos y abdominales, y la misma estructura del palpo maxilar externo. Por este último carácter, estas 2 especies se sitúan en una condición primitiva por tener un palpo simple con 4 sedas sublobulares, que es la condición plesiomórfica de la familia Isotomidae (Fjellberg, 1984). Proisotoma turikana se caracteriza por la reducción del número de ojos, por un mayor número de sensilas accp en los terguitos, acercándose al tipo Isotominae primitivo con un revestimiento sensilar abundante (Deharveng, 1979), y por la distribución de sedas en el dente. Las principales diferencias entre la nueva especie y las próximas se indican en el Cuadro 1.

Material tipo. Holotipo hembra (en preparación), Venezuela, Estado de Zulia, Sierra de Perijá, Mesa Turik, Cueva de la Pared del Norte, guano de guácharos (Steatornis caripensis), 11 de marzo de 1991, leg. J.I. Calvo (LESM). Paratipos: misma localidad y fecha que el holotipo, 5 ejemplares en preparación (LESM), 7 ejemplares (6 hembras y 1 macho) en preparación (colección particular 
de Arbea).

Otro material examinado. Venezuela, Estado de Zulia, Sierra de Perijá, Mesa Turik, Cueva de los Guácharos, guano de guácharos, 15 de marzo de 1991, leg. J.I. Calvo, 1 hembra en preparación (colección particular de Arbea).

Proisotoma santosorum sp. nov. (Figs. 9-13)

Longitud 0, $75 \mathrm{~mm}$. Coloración azul-grisácea. Cuerpo alargado, de la misma forma que $P$. minuta. Revestimiento dorsal y ventral constituido por sedas ordinarias subiguales cortas, lisas y puntiguadas; con macrosedas apenas diferenciadas en los terguitos abdominales V y VI; sensilas poco diferentes de las sedas ordinarias, son un poco más cortas y finas; microsensila del terguito torácico II aparentemente ausente (Fig. 9). Antenas un poco más largas que la diagonal cefálica. Artejo antenal I con 12 sedas ordinarias, 1 microseda ventral y 2 sensilas bastoniformes ventrales, 1 larga y otra corta. Antenal II con 18 sedas ordinarias, 3 microsedas basales y 1 sensila bastoniforme ventrodistal. Antenal III con 23 sedas ordinarias y 1 órgano sensorial formado por 2 microsensilas internas redondeadas, 2 sedas sensoriales de guardia largas y finas y 2 microsensilas ventroexternas. Revestimiento del antenal IV abundante, formado por sedas ordinarias y 15-17 sedas sensoriales, un poco más cortas y gruesas que las sedas ordinarias; apicalmente presenta 1 microseda y un organito muy pequeño; sin vesícula apical exértil (Fig. 10). Cabeza con 6+6 ojos, dentro de 1 mancha ocular pigmentada. Órgano postantenal ovalado, 2, 5-3 veces más largo que el diámetro de una corneola (Fig. 11). Palpo maxilar simple, con 3 sedas sublobulares. Quetotaxia clipeolabral 3/ 5, 5, 4; margen anterior del labro sin estructuras particulares.

Quetotaxia sensorial sobre los terguitos:

\begin{tabular}{llllllll} 
& \multicolumn{1}{c}{ Tórax } & \multicolumn{9}{c}{ Abdomen } \\
Sensila & II & III & I & II & III & IV & V \\
accp & 1 & 1 & 2 & 2 & 2 & 2 & 4 \\
al & 2 & 2 & 0 & 0 & 0 & 0 & 0
\end{tabular}

Revestimiento ordinario abundante. Terguito abdominal VI con las sedas dorsales impares $a 0, m 0 \mathrm{y}$ p0. Ventralmente se observan: $3+3$ sedas a lo largo de la línea ventral cefálica; esternitos torácicos I, II, III sin sedas; tubo ventral con 3-4 sedas posterobasales y 4+4 sedas laterodistales; abdominal II sin sedas centrales. El esternito abdominal IV lleva 8-10 sedas en la subcoxa anterior y 5-6 en la subcoxa posterior (Fig. 9). Fúrcula corta; manubrio con 12-13 pares de sedas posteriores y $1+1$ sedas anteriores más gruesas; dente no anillado, con

Cuadro 1. Principales caracteres morfológicos de las nuevas especies neotropicales de Proisotoma y las más próximas

\begin{tabular}{|c|c|c|c|c|c|c|}
\hline & P.minuta & P. subminuta & P. minima & P. oliveirae & $\begin{array}{l}\text { P. santosorum } \\
\text { sp. nov. }\end{array}$ & $\begin{array}{l}\text { P. turikana } \\
\text { sp. nov. }\end{array}$ \\
\hline Número de ojos & $8+8$ & $7+7$ & $5+5$ & $6+6$ & $6+6$ & $5+5$ \\
\hline OPA/ diámetro corneola & 2,5 & $2-2,5$ & 2,5 & 1 & $2,5-3$ & 2,5 \\
\hline $\begin{array}{l}\text { Número de sedas ventrales: } \\
\text { Sobre torácico II } \\
\text { sobre torácico III } \\
\text { sobre abdominal II }\end{array}$ & $\begin{array}{c}0 \\
1+1-2 \\
\text { varias }\end{array}$ & $\begin{array}{l}0 \\
0 \\
0\end{array}$ & $\begin{array}{l}0 \\
0 \\
?\end{array}$ & $\begin{array}{l}1+1 \\
2+2 \\
3-4\end{array}$ & $\begin{array}{l}0 \\
0 \\
0\end{array}$ & $\begin{array}{c}0 \\
1+1 \\
3+3\end{array}$ \\
\hline $\begin{array}{l}\text { Número de sedas en el Dente: } \\
\text { anteriormente } \\
\text { posteriormente }\end{array}$ & $\begin{array}{l}6(1,2,3) \\
6(3,2,1)\end{array}$ & $\begin{array}{l}6(1,2,2,1) \\
6(3,2,1)\end{array}$ & $\begin{array}{l}5-6(1,2,2-3) \\
\quad 6(3,2,1)\end{array}$ & $\begin{array}{c}5(2,3) \\
5(2,2,1)\end{array}$ & $\begin{array}{l}6(1,2,3) \\
5(2,2,1)\end{array}$ & $\begin{array}{l}6(1,2,2,1) \\
7(4,2,1)\end{array}$ \\
\hline Relación dente/ mucrón & $4 / 1$ & $4,3 / 1$ & $2,3 / 1$ & $2,6 / 1$ & $3 / 1$ & $3,7 / 1$ \\
\hline $\begin{array}{l}\text { Número de sedas sublobulares del } \\
\text { palpo maxilar externo }\end{array}$ & 4 & 3 & 1 & $?$ & 3 & 4 \\
\hline Número de dientes del tenáculo & $4+4$ & $4+4$ & $3+3$ & $4+4$ & $3+3$ & $4+4$ \\
\hline Sedas capitadas del tibiotarso & $(1,1,1)$ & $0,0,0$ & $0,0,0$ & $(1,1,1)$ & $0,0,0$ & $1,1,1$ \\
\hline $\begin{array}{l}\text { Sensilas dorsales (Fig. 3): } \\
\text { al del torácico III } \\
\text { accp del torácico II } \\
\text { accp de abdominal I-IV }\end{array}$ & $\begin{array}{l}1 \\
1 \\
2\end{array}$ & $\begin{array}{l}1 \\
1 \\
2\end{array}$ & & $\begin{array}{l}1 \\
1 \\
2\end{array}$ & $\begin{array}{l}2 \\
1 \\
2\end{array}$ & $\begin{array}{l}2 \\
2 \\
3\end{array}$ \\
\hline
\end{tabular}


6 sedas en la cara anterior ( 1 basal, 2 mediales y 3 en la parte distal) y 5 sedas en la cara posterior ( 2 sedas basales, 2 submediales y 1 subapical) (Fig. 12); relación manubrio/ dente/ mucrón: 6/3/ 1; mucrón tridentado. Tenáculo con $3+3$ dientes y 1 seda en el cuerpo. Ungues corto, sin dientes internos ni laterales, siendo la relación longitud de su borde interno: anchura de la base =2.4-2.6. Apéndice empodial con una lamela interna redondeada, bien desarrollada y sin dientes, alcanza el 55-65\% de la longitud del borde interno de la uña. Tibiotarsos sin espolones mazudos; las sedas se disponen en 3 verticilos de 7 sedas cada uno, en las patas I y II; la disposición de las sedas en el tibiotarso III se indica en la figura 13.
Quetotaxia de las patas:

Subcoxa 1 Subcoxa 2 Coxa Trocánter

Pata I

Pata II

0

Pata III

1

1

4-5

4

$4-5$

4

9

8

8

Fémur

\section{Tibiotarso}

15

21

14

$13-15$
21

25

\section{Clave para las especies neotropicales del complejo Proisotoma}

1. Mucrón con 4 dientes .2

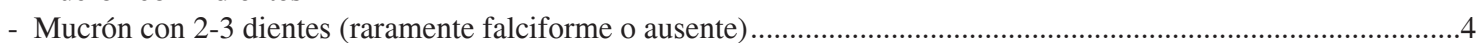

2. Con $8+8$ ojos

- Con 6+6 ojos

Proisotoma andina Rapoport y Rubio, 1968

3. Órgano postantenal aproximadamente tan largo como la diagonal de un ojo. Dente con 6 sedas posteriores y 7 anteriores Proisotoma pajonalica Loksa y Rubio, 1966

- Órgano postantenal aproximadamente 1, 5 veces la diagonal de un ojo. Dente con 5 sedas posteriores y 10 anteriores Proisotoma lutii Izarra, 1973

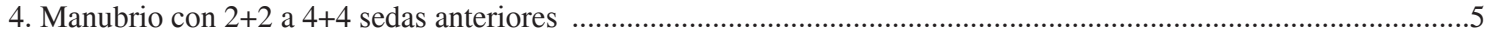

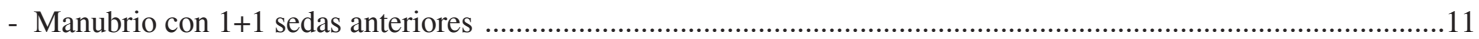

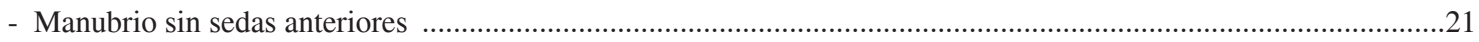

5. Con $8+8$ ojos. Mucrón con 2 dientes y sin lamelas laterales, o con lamelas poco diferenciadas .............................6

- Con un número reducido de ojos. Mucrón con 3 dientes y sin lamelas ..................................................................10

6. Uñas con diente interno ........................................................................................................... Ssotoma immersa Folsom, 1924

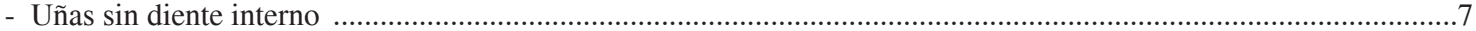

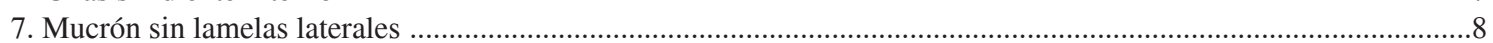

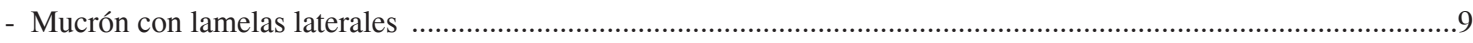

8. Dente con 8-10 sedas posteriores y unas 23 anteriores Isotoma tenella Reuter, 1895

- Dente con 14-22 sedas posteriores y 47-55 anteriores Proisotoma biseta Rapoport, 1963

9. Dente con unas 12 sedas posteriores y unas 50 anteriores ..Proisotoma koepckei Winter, 1967

- Dente con unas 18 sedas posteriores y 30-40 anteriores Proisotoma paronai Börner, 1907

10. Con $6+6$ ojos

Sin ojos o con $1+1$ ojos beta Christiansen y Bellinger, 1980

11. Mucrón con 3 (a veces 2) dientes y sin lamelas laterales. Dente con 4-7 sedas anteriores que se extienden hasta la base: Proisotoma y especies afines......................................................................................................................12

- Mucrón con 2 (a veces 3) dientes y generalmente con lamelas laterales. En el dente las sedas anteriores se agrupan en la parte distal, no presentando sedas en la parte basal: Scutisotoma y especies afines

- Mucrón falciforme. Dente con una seda anterior distal y 2 sedas posteriores basales

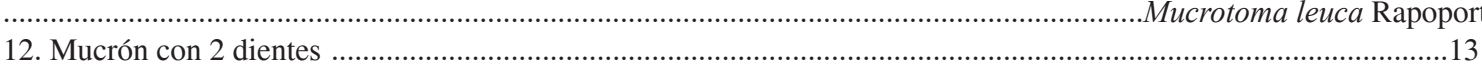

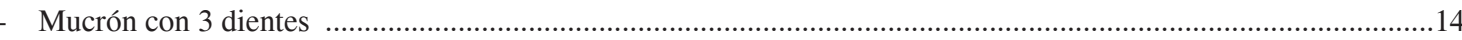

13. Con $6+6$ ojos. Dente con 8 sedas anteriores (11123 desde la base hacia el ápice).

Varisotoma alticola Loksa y Rubio, 1966

- Con $2+2$ ojos. Dente con (8)-9 sedas anteriores (111222). Proisotoma ramosi Arlé, 1959

14. Con $8+8$ ojos. Dente con 6 sedas anteriores (123)

- Con 7+7 ojos. Dente con 6 sedas anteriores (1221). Proisotoma minuta (Tullberg, 1871)

- Con 6+6 ojos . Proisotoma subminuta Denis, 1931 Con $5+5$ ojos . .15

15. Dente con 5 sedas anteriores (23) y 5 posteriores (221) 16 Proisotoma oliveirae Deharveng, 1984 
- Dente con 5 sedas anteriores (122) y 7 posteriores (322)

Proisotoma micrura Börner, 1907

Dente con 6 sedas anteriores (222) y 3 posteriores (21)

Proisotoma intermixta Handschin, 1928

Dente con 6 sedas anteriores (123) y 5 posteriores (221)

..Proisotoma santosorum sp. nov.

16. Dente con 5-6 sedas anteriores (122-3) y 6 posteriores (321)

Proisotoma minima (Absolon, 1901) Dente con 6 sedas anteriores (1221) y 7 posteriores (421)...

Proisotoma turikana sp. nov.

17. Mucrón con 3 dientes y lamelas laterales. Con $8+8$ ojos. Dente con 22-24 sedas anteriores que llegan casi hasta la parte basal y 14-15 sedas posteriores.

Scutisotoma titusi (Folsom, 1937)

Mucrón con 2 dientes 18

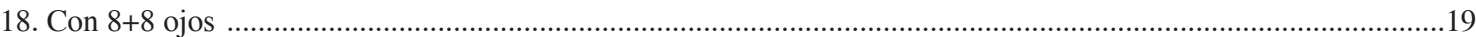

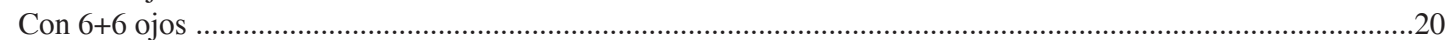

Con $5+5$ ojos. Dente con 1 seda anterior distal y 2 sedas posteriores basales ...........................Proisotoma intermedia Izarra, 1969

19. Mucrón con lamelas laterales. Dente con 4 sedas anteriores distales y 5-9 sedas posteriores.

Proisotoma ridleyi Denis, 1933

Mucrón sin lamelas. Dente con 4-5 sedas anteriores distales y 5-6 sedas posteriores

Proisotoma bulba Christiansen y Bellinger, 1980

20. Mucrón con lamelas laterales reducidas. Dente con 1 seda anterior distal y 8 sedas posteriores........

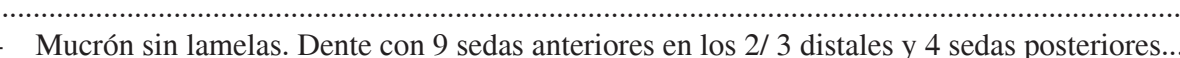

Proisotoma perparva Jackson, 1927

Proisotoma macedoi Winter, 1967

21. Mucrón generalmente masivo y con lamelas laterales bien diferenciadas. Cuerpo relativamente corto; con un perfil redondeado entre los abdominales 4 y 6

Mucrón, cuando está presente, con 1-2 dientes y generalmente sin lamelas laterales, o con lamelas reducidas.

- Cuerpo largo y cilíndrico; visto de perfil presenta una inflexión brusca entre los abdominales 4 y 5 . Dente con no más de 3

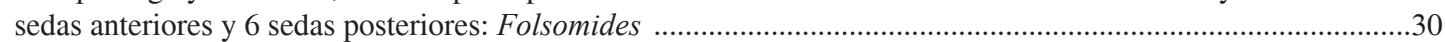

22. Dente normalmente con más de 3 sedas anteriores y 6 posteriores: Ballistura y especies afines .........................23

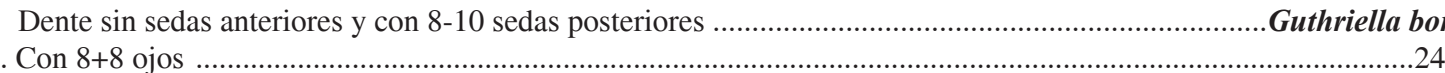

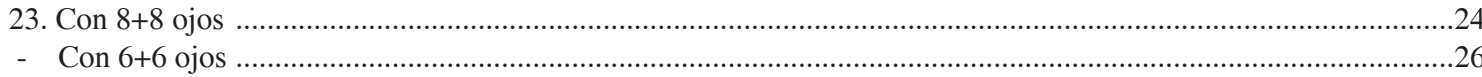

Con $5+5$ ojos. Dente con 2 sedas anteriores distales y 7 sedas posteriores

Proisotoma plicicauda Handschin, 1927

Con 1-2+1-2 ojos. Dente con 1-2 sedas anteriores distales y 9 sedas posteriores

Proisotoma troglobia Rapoport y Maño, 1969

24. Dente con 1 seda anterior distal y con 7-8 sedas posteriores

Ballistura fitchi (Denis, 1933)

Dente con numerosas sedas anteriores y posteriores 25

25. Órgano postantenal aproximadamente tan largo como la diagonal de un ojo. Mucrón ancho, en forma de canoa.

Ballistura schoetti (Dalla Torre, 1895)

Órgano postantenal aproximadamente el doble de largo que la diagonal de un ojo. Mucrón más corto

Isotoma obtusicauda (Schäffer, 1897)

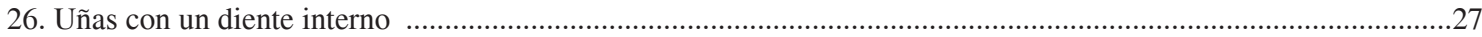

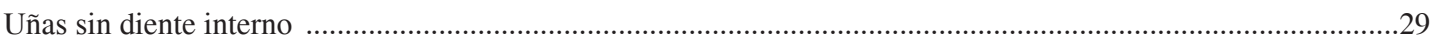

27. Dente con 1 seda anterior distal y 13-15 sedas posteriores

Proisotoma fatonei Rapoport, 1959

Dente con 2-3 sedas anteriores distales

.28

28. Dente con 9-15 sedas posteriores. Órgano postantenal 2, 7 veces el diámetro de un ojo

Ballistura laticauda (Folsom, 1937)

Dente con 16-17 sedas posteriores. Órgano postantenal 1, 5 veces el diámetro de un ojo

29. Dente con 2 sedas anteriores distales y 17-19 sedas posteriores

Ballistura filifera (Denis, 1931)

Dente con 5 sedas anteriores distales y $12-15$ sedas posteriores

Proisotoma fernandeziana Rubio, 1974

30. Con 1-2 pares de ojos. Órgano postantenal estrecho y alargado. Dente sin seda anterior. Tenáculo sin sedas.

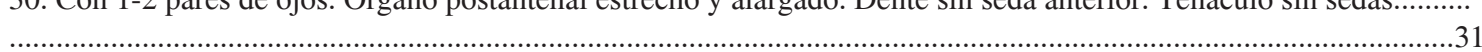

Con 4+4 o más ojos. Órgano postantenal ovalado. Tenáculo con una seda...

\section{4}

31. Dente con 3 sedas posteriores. Tenáculo con $3+3$ dientes. Con 1-2 pares de ojos

Dente con 1 o 2 sedas posteriores. Tenáculo con $2+2$ dientes. Con $2+2$ ojos

Folsomides parvulus Stach, 1922 .32

32. Dente con 1 seda posterior

Dente con 2 sedas posteriores

33. Con mucrón

Folsomides monosetis Massoud y Rapoport, 1968

Sin mucrón

Folsomides semiparvulus Fjellberg, 1993 ..Folsomides yucatanicus Kovac y Palacios-Vargas, 1996 


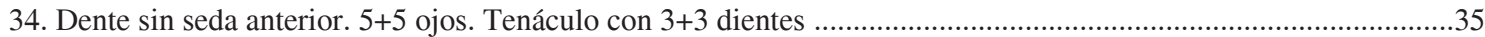

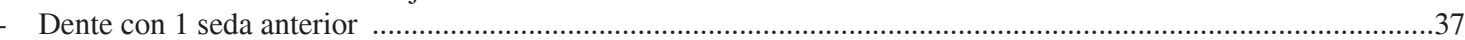

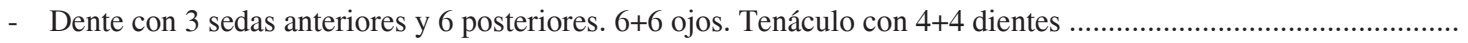

Folsomides centralis (Denis, 1931)

35. Dente con 2 sedas posteriores Folsomides chichinautzini Kovac y Palacios-Vargas, 1996

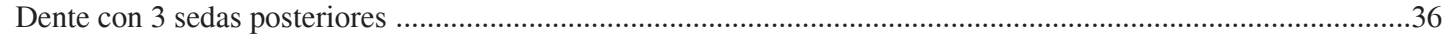

36. Con mucrón. A veces sólo dos sedas posteriores en el dente .................................................. Folsomides marchicus (Frenzel, 1941)

Sin mucrón Folsomides andinensis Díaz y Najt, 1984

37. Con $4+4$ ojos. Dente con 2 sedas posteriores. Tenáculo con $3+3$ dientes

Folsomides socorrensis Kovac y Palacios-Vargas, 1996

- Con 5+5 ojos. Dente con 3 sedas posteriores (raramente 2). Tenáculo con 4+4 dientes

Folsomides angularis (Axelson, 1905)

Con $8+8$ ojos. Dente con 3 sedas posteriores. Tenáculo con 3+3 dientes (Nota: la posición de esta especie en Folsomides es incierta ya que tiene los dos últimos segmentos abdominales fusionados).............Folsomides teres Christiansen y Bellinger, 1980.

Etimología. Se dedica esta especie a la familia de Homero Santos Santos (Homero, Juan, Delia, Josefa, Laura y Aurora), por su apoyo en la colecta de colémbolos en las grutas de Bustamante, Nuevo León.

\section{Comentarios taxonómicos}

Esta especie fue citada como Proisotoma sp. nov. como P. ca. minima (Palacios-Vargas, 1991) de la localidad tipo. Al igual que la especie anterior, $P$. santosorum sp. nov. pertenece al mismo grupo de especies que $P$. minuta. La especie más próxima es $P$. subminuta por presentar el mismo número de sedas en los esternitos torácicos y abdominales, y la misma estructura del palpo maxilar externo. Por este último carácter, estas 2 especies se sitúan en una condición bastante primitiva por tener un palpo simple con 3 sedas sublobulares. Proisotoma santosorum se caracteriza por la reducción del número de ojos, por la distribución de sedas en el dente, y por el número de dientes del tenáculo. Las principales diferencias entre la nueva especie y las más próximas se indican en el Cuadro 1.

Material tipo. Holotipo hembra (en preparación), México, Estado de Nuevo León: Grutas de Bustamante; ex madera en descomposición, 28 de marzo de 1986, J. Palacios col. (LESM). Paratipos: misma localidad y fecha que el holotipo, 2 machos y 2 hembras (en preparación) (LESM).

Otro material examinado. México, Estado de Nuevo León: Grutas de Bustamante; ex madera en descomposición, 2 de febrero de 2005, D. Estrada y J. Palacios cols. 7 ejemplares (LESM).

Especies neotropicales del complejo relacionado con Proisotoma.

Potapov et al. (2006) definen el complejo de especies relacionadas con Proisotoma por 2 caracteres morfológicos clásicos: reducción variable de la fúrcula y ninguna o pocas sedas anteriores en el manubrio. Siguiendo esta definición, la fauna de colémbolos neotropicales incluiría en este complejo, además de las especies de Proisotoma citadas en la introducción, otras 7 especies distribuidas en 4 géneros (Mari Mutt y Bellinger, 1990): Folsomides Stach, 1922: F. andinensis Díaz y Najt, F. angularis (Axelson), F. monosetis Massoud y Rapoport y $F$. parvulus Stach (=F. americanus Denis); Ballistura Börner, 1906: B. schoetti (Dalla Torre); Mucrotoma Rapoport y Rubio, 1963: M. leuca Rapoport y Rubio; y Bonetrura Christiansen y Bellinger, 1980: B. boneti (Yosii). En esta región se han citado con posterioridad otras 6 especies de Folsomides: F. marchicus (Frenzel) (Palacios-Vargas y Castaño-Meneses, 2002), F. teres Christiansen y Bellinger (Vázquez y Palacios-Vargas, 2004), F. yucatanicus Kovac y Palacios-Vargas, F. socorrensis Kovac y PalaciosVargas, F. chichinautzini Kovac y Palacios-Vargas y $F$. semiparvulus Fjellberg (Kovac y Palacios-Vargas, 1996), y una especie de Ballistura: B. laticuada (Folsom) (PalaciosVargas y Gómez-Anaya, 1994). Algunas de estas especies pertenecen claramente a géneros redefinidos recientemente con base en caracteres quetotáxicos (quetotaxia tibiotarsal, del cono bucal, número y disposición de las macrosensilas y microsensilas tergales, desarrollo de la fúrcula por Fjellberg (1993) (Folsomides) y por Potapov (2001) y Potapov et al. (2006) (Ballistura, Proisotoma, Scutisotoma); estas especies se señalan en la clave con negritas. En otras especies, estos caracteres diagnósticos en el nivel de género no son conocidos, por lo que no se pueden incluir con claridad en estos géneros, o incluso sería necesario establecer unos nuevos para alguna de ellas. Por este motivo, hasta que se efectúe una revisión de estas especies conforme a los nuevos caracteres morfológicos considerados actualmente, hemos preferido referirlas en la siguiente clave con el binomio que se les asignó en la descripción original. 


\section{Agradecimientos}

Al Grupo Espeleológico Satorrak, a la Unión de Espeleólogos Vascos UEV.EEE y a la Sociedad Venezolana de Espeleología, cuyo trabajo de prospección en la Mesa Turik permitió recolectar la nueva especie de Venezuela. A Juan Santos Gracia y Raúl Capistrán Gracia, la expedición a las grutas de Bustamantes, Nuevo Léon, donde se recolectó la nueva especie mexicana de Proisotoma. Ada Alicia Ruiz Castillo colaboró en la entintada de los dibujos y preparación del manuscrito final.

\section{Literatura citada}

Cutz-Pool, L. Q., J. G. Palacios-Vargas, G. Castaño-Meneses y N. E. García-Calderón. 2007. Edaphic Collembola from two agroecosystems with contrasting irrigation type in Hidalgo State, Mexico. Applied Soil Ecology 36:46-52.

Deharveng, L. 1979. Chétotaxie sensillaire et phylogenese chez les collemboles Arthropleona. Travaux du Laboratoire d'Écobiologie des Arthropodes Édaphiques, Toulouse 1:115.

Fjellberg, A. 1984. The maxillary outer lobe, an important systematic tool in Isotomidae (Collembola). Annales de la Société Royale Zoologique de Belgique 114:83-88.

Fjellberg, A. 1993. Revision of European and North African Folsomides Stach with special emphasis on the Canarian fauna (Collembola: Isotomidae). Entomologica Scandinavica 23:453-473.

Kovac, L. y J. G. Palacios-Vargas. 1996. A survey of Mexican Folsomides (Collembola: Isotomidae) with description of three new species. European Journal of Entomology 93:595606.

Mari Mutt, J. A. y P. F. Bellinger. 1990. A Catalog of the
Neotropical Collembola. Including Nearctic areas of Mexico. Flora \& Fauna Handbook 5, Sandhill Crane, Gainesville, Florida. 237 p.

Palacios-Vargas, J. G. 1990. Nuevos Collembola del estado de Chihuahua, México. Folia Entomológica Mexicana 79:5-32.

Palacios-Vargas, J. G. 1991. La fauna de las grutas de Nuevo León, México. Mundos Subterráneos 2: 6-12.

Palacios-Vargas, J. G. y J. A. Gómez-Anaya. 1994. Los Collembola (Hexapoda: Apterygota) de Chamela, Jalisco, México (distribución ecológica y claves). Folia Entomológica Mexicana 89:1-34

Palacios-Vargas, J. G., G. Castaño-Meneses y B. E. MejíaRecamier. 2000. Collembola. In Biodiversidad, taxonomía y biogeografía de artrópodos de México: hacia una síntesis de su conocimiento, vol. II, J.E. Llorente, E. González y N. Papavero (eds.). Comisión Nacional para el Conocimiento y Uso de la Biodiversidad/Universidad Nacional Autónoma de México, México, D.F.. p. 249-273.

Palacios-Vargas, J. G. y G. Castaño-Meneses. 2002. Collembola associated with Tillandsia violacea (Bromeliaceae) in Mexican Quercus-Abies forests. Pedobiologia 46: 395-403.

Potapov, M. 2001. Isotomidae. In Synopses on Palaearctic Collembola, vol. 3, W. Dunger (ed.). Abhandlungen und Berichte des Naturkundemuseums Görlitz 73:1-603.

Potapov, M., A. Babenko y A. Fjellberg. 2006. Taxonomy of the Proisotoma complex. Redefinition of genera and description of new species of Scutisotoma and Weberacantha (Collembola, Isotomidae). Zootaxa 1382:1-74.

Vázquez, M. M. y J. G. Palacios-Vargas. 2004. Catálogo de Colémbolos (Hexapoda: Collembola) de Sian Ka'an, Quintana Roo, México. Comisión Nacional para el Conocimiento y Uso de la Biodiversidad/Universidad de Quintana Roo, Chetumal, Quintana Roo.123 p.

Viloria, A., F. Herrera y C. Galán. 1992. Resultados preliminares del estudio del material biológico colectado en Turik. Karaitza 1: 29-30. 